\title{
Imaging Soil Bacteria in an Environmental Scanning Electron Microscope
}

\author{
J.L. Saleta, and P. Holden
}

Donald Bren School of Environmental Science and Management, University of California, Santa Barbara, CA 93106

The introduction of the Environmental Scanning Electron Microscope (ESEM) has made possible, with little or no specimen preparation, the observation of the biotic constituents of soil and their spatial relation with the abiotic constituents. The capability of this instrument to operate at a water vapor pressure over 4.6 Torr (611 pascals), the vapor pressure of water at $0^{\circ} \mathrm{C}$, makes it feasible to image a saturated specimen, and document the processes of hydration and dehydration [1]. We have used this capability to observe a simplified soil model, a culture of bacteria (Pseudomonas aeruginosa) on clean quartz sand. Our objective is to optimize the observation conditions to be used in the future when addressing more complex systems.

The soil model was mounted on a temperature-controlled Peltier stage provided for the FEI Co. XL30 ESEM FEG, and imaged at $5 \mathrm{KeV}$ to minimize beam damage and reduce beam penetration into the cells while keeping an acceptable signal to noise ratio. The sample relative humidity plays a crucial role in the capacity to distinguish the soft biotic phase (bacteria and their exopolymeric substances or EPS) from the hard mineral phase (quartz). We found that keeping the specimen close to a fully hydrated state, without the presence of standing water, provides the best differentiation between both phases, as can be seen in Figures 1 and 2. Figure 1 shows several cells on the surface of a sand grain right after the dehydration process started. Figure 2 shows the same area several minutes later, well into the dehydration process. Microbes are bound to the substrate and to each other by a layer of hydrated EPS that can be responsible for some degree of water retention that would facilitate the differentiation of the cells or biofilms from the mineral substrate. In hydration/dehydration experiments we have observed higher water retention in the areas colonized by bacteria. Figure 3 shows a sand grain partially colonized by bacteria. After completely hydrating the specimen and beginning the dehydration process, the areas more heavily colonized are still retaining water, while the uncolonized surrounding areas have dried out (Figure 4). In many instances, when observing soil specimens it is difficult to determine whether a rod-shaped feature is a microbe or part of the mineral substrate. "Electronic etching", or concentrating the beam in part of the rod can often give us the answer, given the higher sensitivity of the softer cell material to the electron beam as compared to the harder mineral substrate. Figure 5 and 6 show a bacterial colony before and after the beam was concentrated in the upper-right area, showing severe damage in the biotic phase and almost no disturbance in the mineral phase. These techniques together, i.e. differentiation on the basis of water retention and on the basis of susceptibility to beam etching, represent improved strategies for discovering bacteria against the complex background of the soil matrix.

\section{References}

[1] J.I. Goldstein et al., Scanning Electron Microscopy and X-ray Microanalysis, Plenum, New York, 1992.

[2] This research was supported by the National Science Foundation BES-99772 


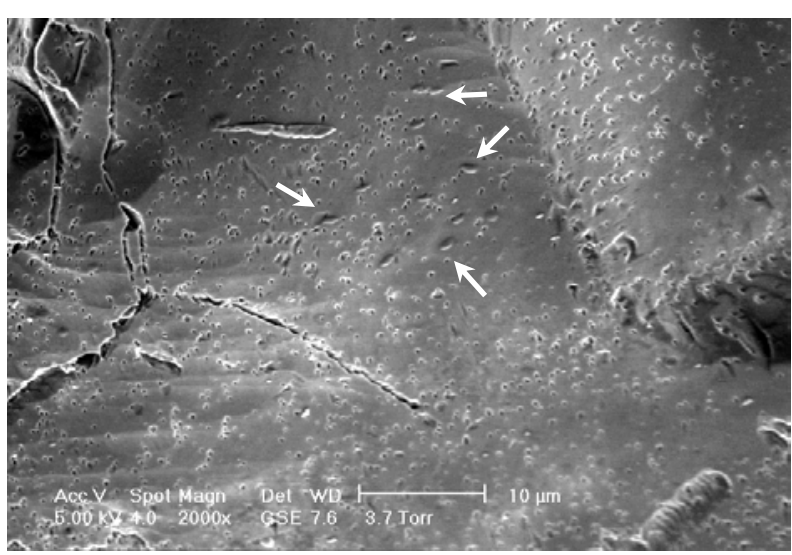

Fig. 1. Bacteria on sand at the beginning of the dehydration process

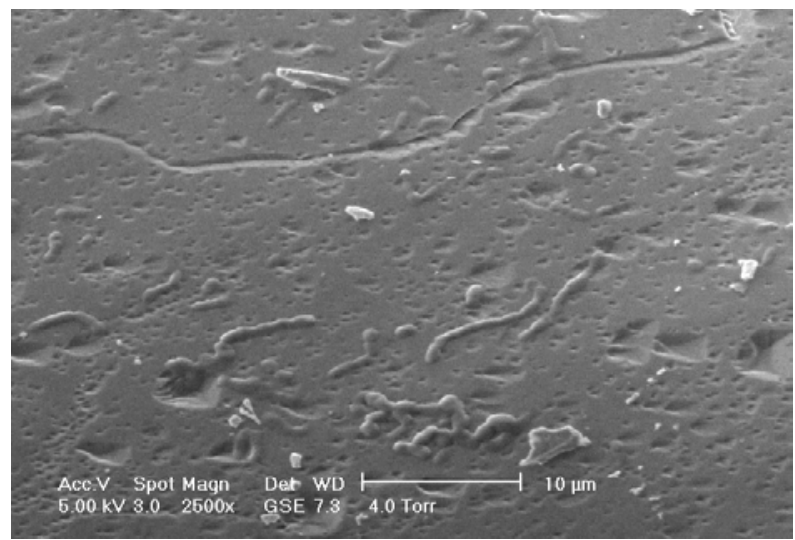

Fig. 3. Bacteria partially colonizing a sand grain, slightly under dew point.

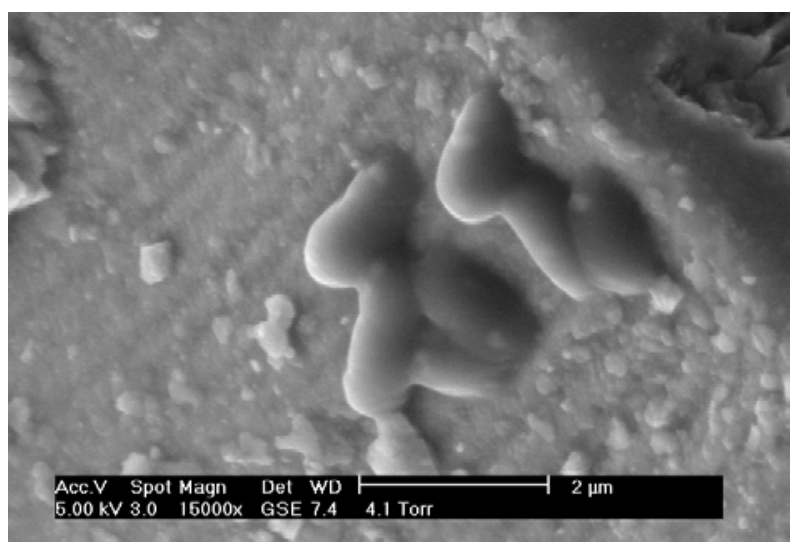

Fig. 5. Colony of bacteria

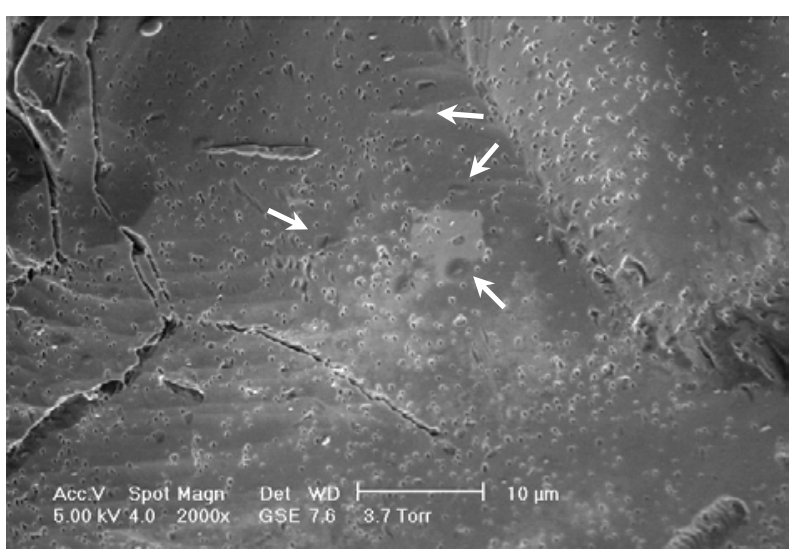

Fig. 2. Same area shown in Fig.1 a few minutes into the dehydration process

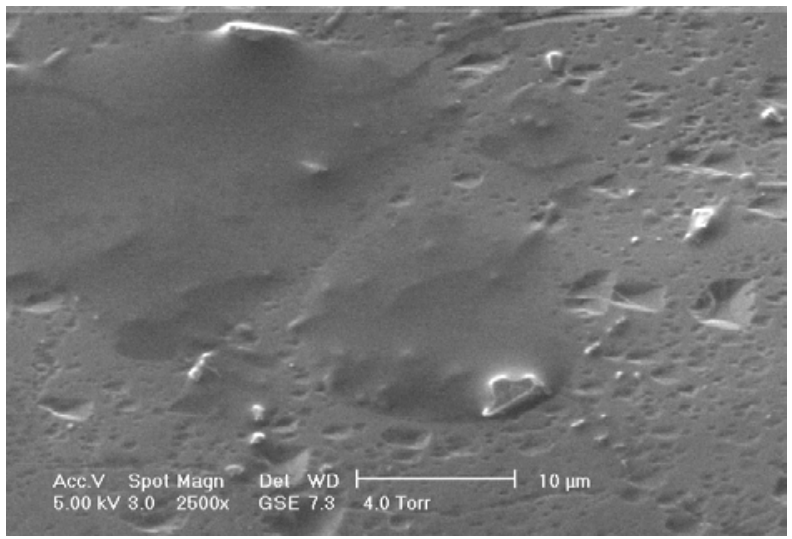

Fig. 4. Same area shown in Fig.3, after full hydration and starting to dehydrate. Notice the water being retained primarily in the colonized areas.

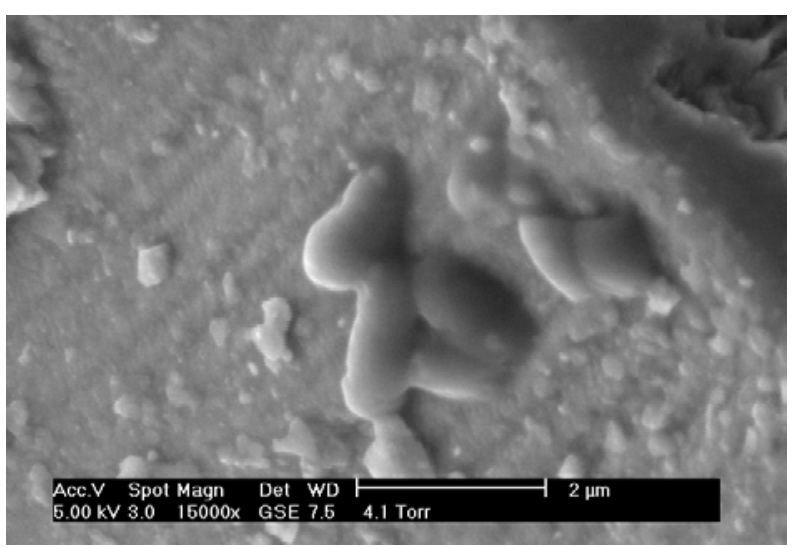

Fig. 6. Same colony of Fig.5 after electronic etching at the upper right corner 